\title{
Negative Magnetoresistance in Dual Spin Valve Structures With a Synthetic Antiferromagnetic Free Layer
}

\author{
C. Fowley, B. S. Chun, and J. M. D. Coey \\ CRANN, School of Physics, Trinity College, Dublin, Ireland
}

\begin{abstract}
Giant magnetoresistance (GMR) in spin valves is due to spin-dependent scattering occurring at ferromagnet/normal metal (F/N) interfaces and/or in the ferromagnetic layers. In a spin valve with a typical $\mathrm{F} / \mathrm{N} / \mathrm{F}$ structure where the spin scattering asymmetry factor $(\alpha)$ of both $\mathrm{F} / \mathrm{N}$ interfaces is the same (more or less than 1), the GMR is expected to be positive. If $\alpha$ is greater than one at one $\mathrm{F} / \mathrm{N}$ interface and less than one at the other $\mathrm{F} / \mathrm{N}$ interface, however, the GMR is expected to be negative. Here, we show that the F1/Cu/SAF/Cu/F2/IrMn dual spin valve structure exhibits negative GMR, where F1 and F2 are CoFe and SAF $=\mathrm{CoFe} / \mathrm{Ru} t / \mathrm{CoFe}$, due to both opposite electron spin scattering asymmetry factor at the $\mathrm{CoFe} / \mathrm{Ru} / \mathrm{CoFe}$ interfaces as well as the electrical separation of the overall structure into two GMR spin valves connected in parallel. A GMR of $6 \%$ is observed in the structure without the Ru spacer layer, insertion of a $0.6 \mathrm{~nm}$ thick $\mathrm{Ru}$ in the SAF results in a negative GMR ratio of $-3 \%$, which becomes positive again at the $R u$ thickness of $0.8 \mathrm{~nm}$, the oscillation from positive to negative MR is consistent with interlayer exchange coupling period across the Ru spacer.
\end{abstract}

Index Terms-Dual spin valve, negative GMR, synthetic antiferromagnet.

\section{INTRODUCTION}

$\mathbf{S}$ INCE the discovery of giant magnetoresistance (GMR) [1], [2], extensive experimental and theoretical investigations have been performed on spin valve systems because of the wide use of GMR in high density magnetic recording read heads, which is due to their large magnetoresistance (MR) and high MR sensitivity. The basic spin valve (SV) structure is composed of two thin ferromagnetic layers separated by a thin nonmagnetic spacer layer. The GMR effect results from spin dependent scattering of the conduction electrons in the bulk and/or at the interface of the ferromagnetic layers with the nonmagnetic spacer. Its magnitude is related to the spin scattering asymmetry factor of the interface, $\alpha$ (defined by $\alpha=\rho_{\downarrow} / \rho_{\uparrow}$ where $\rho_{\uparrow}\left(\rho_{\downarrow}\right)$ is the resistivity for majority (minority) spin channel [3].

When $\alpha$ at the interfaces in the ferromagnetic (F1)/nonmetal $(\mathrm{N}) /$ ferromagnetic (F2) SV structure is the same and are both larger or smaller than 1, the GMR is expected to be positive (the resistance is larger when the magnetizations are aligned antiparallel and smaller when they are parallel). For a SV with different $\alpha$ at each interface, however, the negative GMR is possible [4], [5]. In the case of negative GMR, the resistance is smaller when the magnetizations of the ferromagnetic layers are antiparallel to each other, and larger in the parallel case.

A negative GMR effect was first reported by George et al. in a $\mathrm{Fe} / \mathrm{Cr} / \mathrm{Fe} / \mathrm{Cu} / \mathrm{Fe}$ multilayer system [4]. The MR, which was negative at low field and positive at high field, resulted from strong majority spin dependent scattering at the $\mathrm{Fe} / \mathrm{Cr}$ (where $\alpha<1$ ) interfaces than at the $\mathrm{Fe} / \mathrm{Cu}$ interfaces (where $\alpha>1$ ). Several experimental and theoretical studies have shown a negative GMR effect with different ferromagnetic and spacer layer combinations [6]-[10]. In the $\mathrm{Fe}_{1-\mathrm{x}} \mathrm{V}_{\mathrm{x}} / \mathrm{Au} / \mathrm{Co}$ system [6] the negative GMR originates from the increase of the effective density of states at the Fermi level for majority spin electrons in $\mathrm{Fe}$ when

Manuscript received October 08, 2008. Current version published May 20, 2009. Corresponding author: C. Fowley (e-mail: fowleyc@ @tcd.ie).

Color versions of one or more of the figures in this paper are available online at http://ieeexplore.ieee.org.

Digital Object Identifier 10.1109/TMAG.2009.2018589 it is alloyed with $\mathrm{V}$. It follows that there is an associated reversal of the spin scattering asymmetry factor from $\alpha<1$ to $\alpha>1$ in the $\mathrm{Fe}_{1-\mathrm{x}} \mathrm{V}_{\mathrm{x}}$ system. In $\mathrm{Co} / \mathrm{Ru} / \mathrm{CoRu}$ system [7], the negative GMR is attributed to the doping effect in CoRu alloy, which reduces the bulk spin-dependent scattering to a value smaller than the interface spin dependent scattering.

In this paper, we demonstrate the dependence of GMR on nonmagnetic interlayer $(\mathrm{Ru})$ thickness in a synthetic antiferromagnetic free layer within a dual spin valve structure. Using this method, a GMR of $6 \%$ is observed in the structure without the Ru spacer layer, insertion of a $0.2 \mathrm{~nm}$ thick Ru layer in the $\mathrm{CoFe}$ free layer is enough to change the sign of GMR. With increasing $t$, the GMR ratio decreases to $-3 \%$ but becomes positive again at the $\mathrm{Ru}$ thickness of $0.8 \mathrm{~nm}$.

\section{EXPERIMENT}

Samples were of the following form; $\mathrm{Si}_{/} \mathrm{SiO}_{2} / \mathrm{Ta} 5 / \mathrm{CoFe}(\mathrm{F} 1)$ 1.5/Cu 2.8/[CoFe 1.3/Ru $t / \mathrm{CoFe} 1.3](\mathrm{SAF}) / \mathrm{Cu} 2.8 / \mathrm{CoFe}(\mathrm{F} 2)$ 2.5/IrMn 10/Ta 5 (thickness in $\mathrm{nm}$ ) where Ru thickness was varied from $0-0.8 \mathrm{~nm}$. Samples were grown with and without IrMn antiferromagnetic pinning layer. $\mathrm{Co}_{90} \mathrm{Fe}_{10}$ and $\mathrm{Ir}_{22} \mathrm{Mn}_{78}$ are the target compositions in atomic percent. Layer thicknesses were chosen after optimization to ensure there was no exchange coupling across the $\mathrm{Cu}$ spacers. Samples were prepared using a "Shamrock" deposition tool with a typical base pressure of less than $2 \times 10^{-7}$ Torr. The magnetic layer easy axes were aligned along the same direction by applying a $10 \mathrm{mT}$ magnetic field during deposition. The magnetic properties and magnetoresistance of the spin valve structure were characterized by using a Quantum Design superconducting quantum interference device (SQUID) and Quantum Design physical property measurement system (PPMS), respectively.

\section{RESUlTS AND DisCUSSION}

In Fig. 1, two different GMR characteristics can be distinguished in magnetotransport curves at room temperature for the dual spin valve structure. We clearly observe an oscillation of 


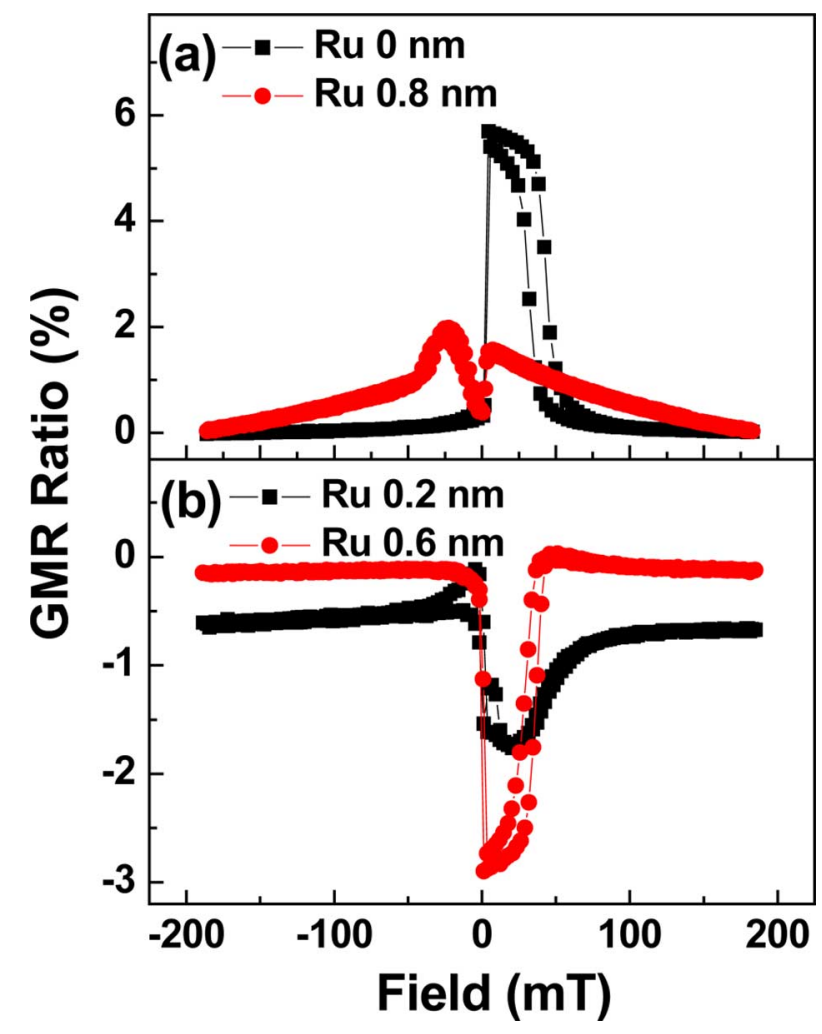

Fig. 1. Ru thickness dependence of the GMR ratio in the Ta $5 / \mathrm{CoFe} 1.5 / \mathrm{Cu}$ 2.8/CoFe 1.3/Ru $t / \mathrm{CoFe} 1.3 / \mathrm{Cu} 2.8 / \mathrm{CoFe} 2.5 / \mathrm{IrMn} 10 / \mathrm{Ta} 5(\mathrm{~nm})$ dual spin valve structure. (a) GMR of $6 \%$ is observed without the Ru spacer layer and with $0.8 \mathrm{~nm}$ of Ru. (b) $0.2 \mathrm{~nm} \mathrm{Ru}$ and $0.6 \mathrm{~nm} \mathrm{Ru}$ samples both show negative GMR.

GMR ratios as a function of Ru thickness up to $0.8 \mathrm{~nm}$ with a change of sign from positive to negative GMR and back again as a function of the Ru layer thickness.

As shown in Fig. 1(a), positive GMR (the resistance is largest for an antiparallel alignment of the magnetization direction) of $6 \%$ is observed without the Ru spacer layer. Upon insertion of a $0.2 \mathrm{~nm}$ Ru layer [Fig. 1(b)], however, between the CoFe layers a negative GMR ratio has been observed.

To explain the negative GMR effect, we have made dual spin valve structures with (Fig. 1) and without (Fig. 2) the IrMn antiferromagnetic exchange bias layer. When the structure is pinned with the IrMn layer (Fig. 1) and the SAF has $0 \mathrm{~nm}$ of Ru the GMR is positive, both unpinned $\mathrm{CoFe}$ free layers ( $\mathrm{F} 1$ and $\mathrm{SAF}$ ) switch close to zero field. The pinned CoFe layer (F2) switches around $30 \mathrm{mT}$. Here $\alpha$ is the same for all interfaces in the system. In contrast, when $0.6 \mathrm{~nm}$ of $\mathrm{Ru}$ is introduced into the SAF a negative GMR, with F1 switching around zero field and the SAF being in the antiparallel state and not switching, is observed. The fixed CoFe layer (F2) again switches around $30 \mathrm{mT}$. In the corresponding sample without antiferromagnetic IrMn layer and a $0.6 \mathrm{~nm}$ Ru spacer, [Fig. 2(b)] the negative GMR is observed, this can be explained by the spin scattering asymmetry factor of the $\mathrm{CoFe} 1.3 / \mathrm{Ru} 0.6 / \mathrm{CoFe} 1.3$ interface being opposite to that in the two outer ( $\mathrm{F} 1$ and $\mathrm{F} 2) \mathrm{CoFe} / \mathrm{Cu}$ interfaces. If the spin scattering asymmetry factor is the same for the F1, F2 and SAF interfaces, either $\alpha>1$ or $\alpha<1$, the resultant GMR ratio is positive.

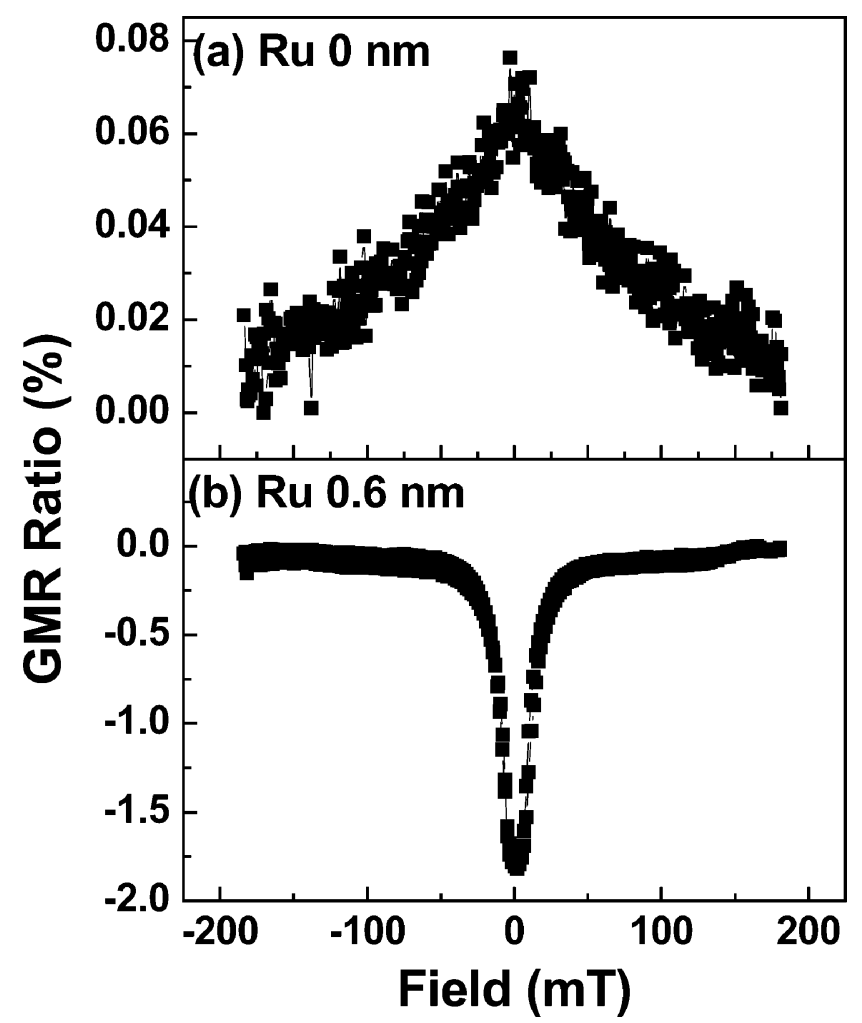

Fig. 2. Ru thickness dependence of the GMR ratio in the case of a (a) $\mathrm{CoFe}$ $2.6(\mathrm{~nm})$, and (b) CoFe 1.3/Ru 0.6/CoFe $1.3(\mathrm{~nm})$ free layer in a dual spin valve structure without an antiferromagnetic IrMn exchange bias layer.

If F1, F2 and SAF interfaces have different spin asymmetry of scattering factor $\left(\alpha_{\mathrm{F} 1,2}>1\right.$ and $\left.\alpha_{\mathrm{SAF}}<1\right)$, the negative GMR ratio is possible [4]. Since the spin scattering asymmetry factor of the two outer $\mathrm{CoFe} / \mathrm{Cu}$ interfaces is $\alpha_{\mathrm{CoFe} / \mathrm{Cu}}>1$, the value of SAF one must be smaller than $1\left(\alpha_{\mathrm{CoFe}} / \mathrm{Ru} / \mathrm{CoFe}<1\right)$.

The value of spin scattering asymmetry may originate from a modification to the density of states at the $\mathrm{CoFe} / \mathrm{Ru} / \mathrm{CoFe}$ interface. Since the Ru has a partially occupied $d$ band at the Fermi energy, which is nearly equal to the Co minority $d$ band, the ferromagnetic $\mathrm{CoFe}$ can induce a magnetic moment on the $\mathrm{Ru}$ atoms near the $\mathrm{CoFe} / \mathrm{Ru} / \mathrm{CoFe}$ interface [7], [11]. As a result, the spin scattering asymmetry at the $\mathrm{CoFe} / \mathrm{Ru} / \mathrm{CoFe}$ interface can be less than 1 . In our dual spin valve structure, since we measure in the current-in-plane (CIP) geometry, most of the current flows through the $\mathrm{Cu}$ spacer layers and as a result the MR originates mainly from the interfaces with the $\mathrm{Cu}$ layers. Essentially it can be treated as two spin valves in electrical parallel (SV-1 and SV-2 in Fig. 4). In the CIP geometry the contribution from the differing $\alpha$ cannot be fully separated from the contributions of the adjacent spin valve channels.

Magnetoresistance measurements have also been performed at low temperature to examine the temperature dependence of the GMR ratio for the films showing negative GMR effects. Low temperature data is shown in Fig. 3. The GMR increases monotonically from less negative to more negative values in the temperature regime from $300 \mathrm{~K}$ to $13 \mathrm{~K}$. The GMR ratios of $\mathrm{CoFe} 1.3 / \mathrm{Ru} 0.2 / \mathrm{CoFe} 1.3$ and $\mathrm{CoFe} 1.3 / \mathrm{Ru} 0.4 / \mathrm{CoFe} 1.3 \mathrm{SAF}$ layered dual spin valve structures were -1.8 and $-2.9 \%$ at $300 \mathrm{~K}$, 


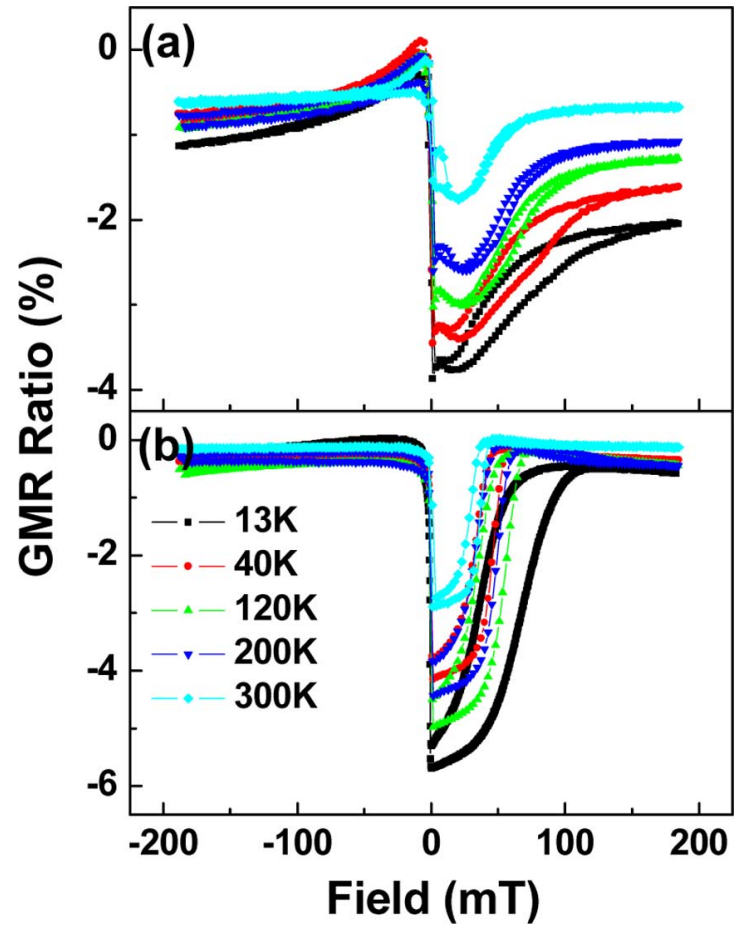

Fig. 3. Temperature dependent GMR ratio for various $\mathrm{SAF}$ free-layered dual spin valve structures: (a) $\mathrm{CoFe} 1.3 / \mathrm{Ru} 0.2 / \mathrm{CoFe} 1.3$, (b) $\mathrm{CoFe} 1.3 / \mathrm{Ru} 0.4 / \mathrm{CoFe}$ $1.3(\mathrm{~nm})$.

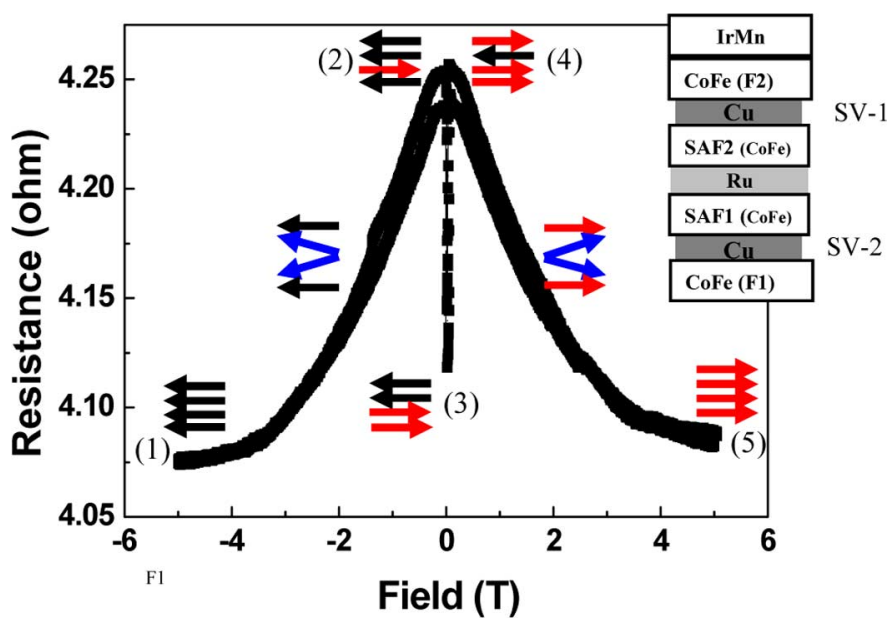

Fig. 4. Magnetization switching behavior of negative GMR dual spin valve structure with $0.6 \mathrm{~nm}$ of Ru.

and -2.9 and $-5.7 \%$ at $13 \mathrm{~K}$, respectively. This can be attributed to the reduction of spin-flip scattering in the dual spin valve layers at low temperature (i.e., at lower temperature the electrons mean free path is longer, and the spin flip scattering within the spacers, both $\mathrm{Cu}$ and $\mathrm{Ru}$, is reduced).

Fig. 4 shows the R-H curve, for a $0.6 \mathrm{~nm}$ Ru layer in the SAF, over a large field range (up to $5 \mathrm{~T}$ ) measured by PPMS. Compared to low field measurements (Figs. 1 and 3), resistance continues to decrease above $200 \mathrm{mT}$ and eventually saturates at $4 \mathrm{~T}$. This slow decrease of resistance at high field is due to antiferromagnetic coupling across Ru in SAF structure and the applied field gradually overcoming the antiferromagnetic exchange coupling. In order to paint a clearer picture of the magnetic moment configuration in high applied field, the magnetization direction of each magnetic layer is represented by arrows in Fig. 4 (with antiferromagnetically coupled CoFe SAF layers. Starting from negative saturation field (1) the magnetization of all the layers are aligned parallel to the field direction. As the field is reduced, the resistance increases gradually, because the magnetization direction of one of the CoFe layers in the SAF free layer rotates towards an antiparallel orientation with respect to that of another SAF layer due to antiferromagnetic interlayer exchange coupling across the Ru layer. [12] The magnetizations of F1 and F2 stay aligned to the applied field until zero field (2). Therefore one of them, in this case (F1), must be antiparallel with respect to one of the SAF layers, in this case the layer adjacent F1. At small positive field the magnetization of $\mathrm{F} 1$ switches (since it is not pinned), this creates a state where the bottom CoFe layer in the SAF is parallel to the CoFe layer (F1) at the bottom of the stack (3). Since the CoFe layers adjacent both $\mathrm{Cu}$ spacers are parallel this is effectively two low resistance spin valves in electrical parallel, SV-1 and SV-2 seperated by Ru, which gives an overall low electrical resistance even though the magnetizations of F1 and F2 are antiparallel. Increasing the field further (4) to the exchange bias of F2 and IrMn causes F2 to switch to the field direction, in this state the top spin valve is in the antiparallel state while the lower spin valve is in the parallel state. As the applied field is increased further (5) to positive saturation the exchange coupling across the SAF is overcome and all layers are aligned parallel.

\section{CONCLUSION}

We clearly observe an oscillation of GMR ratios as a function of Ru thickness from positive to negative values in SAF dual spin valve, as the thickness of the Ru spacer is increased from 0 to $0.8 \mathrm{~nm}$. The sign change of GMR is due to the low resistance parallel configuration of adjacent spin valve channels and the different spin scattering asymmetry factor between the $\mathrm{CoFe} / \mathrm{Ru} / \mathrm{CoFe} \mathrm{SAF}$ structure and the two outer $\mathrm{CoFe} / \mathrm{Cu}(\mathrm{F} 1$ and F2) interfaces. The value of $\alpha_{\mathrm{CoFe}} / \mathrm{Ru} / \mathrm{CoFe} / 1$ originates from a density of states modification at the $\mathrm{CoFe} / \mathrm{Ru} / \mathrm{CoFe}$ interface. The inverted GMR response with synthetic antiferromagnetic free layers has been reported previously [13], [14], however in that case there was only one of the SAF layers contributed to MR (closest the spacer layer). In our cases spin information is obtained from the bottom layer in the SAF due to the dual spin valve geometry. In this dual spin valve configuration it is possible to see a linear MR response from the SAF layer. As a result, we conclude that in this case it is the synthetic antiferromagnetic free layer that creates the inverted MR response around zero field and that the negative GMR is only present when the SAF is antiferromagnetically coupled.

\section{ACKNOWLEDGMENT}

C. Fowley would like to thank Dr. M. Venkatesan for help acquiring the SQUID data. This work was supported by the Science Foundation Ireland as part of the MANSE program. 


\section{REFERENCES}

[1] M. N. Baibich, J. M. Broto, A. Fert, N. Van Dau, F. Petroff, P. Etienne, G. Creuzet, A. Friederich, and J. Chazelas, "Giant magnetoresistance of $(001) \mathrm{Fe} /(001) \mathrm{Cr}$ magnetic superlattices," Phys. Rev. Lett., vol. 61, pp. 2472-2475, 1988.

[2] G. Binasch, P. Grunberg, F. Saurenbach, and W. Zinn, "Enhanced magnetoresistance in layered magnetic structures with antiferromagnetic interlayer exchange," Phys. Rev. B, vol. 39, pp. 4828-4830, 1989.

[3] A. Fert and I. A. Campbell, "Two-current conduction in nickel," Phys. Rev. Lett., vol. 21, pp. 1190-1192, 1968.

[4] J. M. George, L. G. Pereira, A. Barthélémy, F. Petroff, L. Steren, J. L. Duvail, A. Fert, R. Loloee, P. Holody, and P. A. Schroeder, "Inverse spin-valve-type magnetoresistance in spin engineered multilayered structures," Phys. Rev. Lett., vol. 72, pp. 408-411, 1994.

[5] P. Baumgart et al., "Dual Spin Valve Magnetoresistive Sensor," U.S. Patent US5287238, Feb. 15, 1994.

[6] J.-P. Renard, P. Bruno, R. Mégy, B. Bartenlian, P. Beauvillain, C. Chappert, C. Dupas, E. Kolb, M. Mulloy, P. Veillet, and E. Vélu, "Inverse magnetoresistance in the simple spin-valve system $\mathrm{Fe}_{1-x}$ $\mathrm{V}_{x} / \mathrm{Au} / \mathrm{Co}$," Phys. Rev. B, vol. 51, pp. 12821-12823, 1995.

[7] K. Rahmouni, A. Dinia, D. Stoeffler, K. Ounadjela, H. A. M. Van den Berg, and H. Rakoto, "Inverse magnetoresistance in $\mathrm{Co} / \mathrm{Ru} / \mathrm{Co}$ and doped $\mathrm{Co} / \mathrm{Ru} / \mathrm{Co}_{0.92} \mathrm{Ru}_{0.08}$ sandwiches," Phys. Rev. B, vol. 59, pp. 9475-9481, 1999.
[8] D. Z. Yang, B. You, X. X. Zhang, T. R. Gao, S. M. Zhou, and J. Du, "Inverse giant magnetoresistance in $\mathrm{Fe} / \mathrm{Cu} / \mathrm{Gd}_{1-x} \mathrm{Co}_{x}$ spin-valves," Phys. Rev. B, vol. 74, pp. 024411(1)-024411(6), 2006.

[9] O. Bengone, O. Eriksson, S. Mirbt, I. Turek, J. Kudrnovský, and V. Drchal, "Origin of the negative giant magnetoresistance effect in $\mathrm{Co}_{1-x} \mathrm{Cr}_{x} / \mathrm{Cu} / \mathrm{Co}$ (111) trilayers," Phys. Rev. B, vol. 69, pp. 092406(1)-092406(4), 2004

[10] S. van Dijken, X. Fain, S. M. Watts, and J. M. D. Coey, "Negative magnetoresistance in $\mathrm{Fe}_{3} \mathrm{O}_{4} / \mathrm{Au} / \mathrm{Fe}$ spin valves," Phys. Rev. B, vol. 70, pp. 052409(1)-052409(4), 2004.

[11] T. Nozaki, Y. Jiang, Y. Kaneko, A. Hirohata, N. Tezuka, S. Sugimoto, and $\mathrm{K}$. Inomata, "Spin-dependent quantum oscillations in magnetic tunnel junctions with Ru quantum wells," Phys. Rev. B, vol. 70, pp. 172401(1)-172401(4), 2004.

[12] S. S. P. Parkin, N. More, and K. P. Roche, "Oscillations in exchange coupling and magnetoresistance in metallic superlattice structures: $\mathrm{Co} / \mathrm{Ru}, \mathrm{Co} / \mathrm{Cr}$ and Fe/Cr," Phys. Rev. Lett, vol. 64, no. 19, p. 2304, 1990.

[13] T. Nozaki, S. Abe, N. Tezuka, K. Inomata, and S. Sugimoto, "Magnetic switching properties of GMR spin-valves using SyAF free layers," $J$. Jpn. Inst. Metals, vol. 66, no. 11, pp. 1078-1082, 2002.

[14] K. Inomata, T. Nozaki, N. Tezuka, and S. Sugimoto, "Magnetic switching field and giant magnetoresistance effect of multilayers with synthetic antiferromagnet free layers," Appl. Phys. Lett., vol. 81, no. 2, p. 310, 2002. 